\title{
Monotone Comparative Statics in Games with both Strategic Complements and Strategic Substitutes
}

By

\author{
Andrew J. Monaco \\ Department of Economics \\ University of Kansas \\ Lawrence KS, 66045, USA \\ monacoa@ku.edu
}

\author{
Tarun Sabarwal \\ Department of Economics \\ University of Kansas \\ Lawrence KS, 66045, USA \\ sabarwal@ku.edu
}

\begin{abstract}
This paper analyzes games with both strategic substitutes and strategic complements. Such games may behave differently from either games with strategic complements or games with strategic substitutes. In such games, equilibria do not decrease as the parameter increases. Moreover, natural conditions are presented to guarantee that an increase in the parameter leads to an increase in the equilibrium: in other words, conditions to guarantee monotone comparative statics. These conditions are based on intuitive tradeoffs between direct parameter effects and indirect strategic effects. These conditions are needed only for players with strategic substitutes; no conditions are imposed on players with strategic complements. Several examples highlight the results.
\end{abstract}

JEL Numbers: C70, C72

Keywords: Lattice games, strategic complements, strategic substitutes, equilibrium set, monotone comparative statics

First Draft: April 2011

This Version: August 29, 2012 


\section{Introduction}

Games with strategic substitutes (GSS) and games with strategic complements (GSC) formalize two basic economic interactions and have widespread applications. In GSC, best-response of each player is weakly increasing in actions of the other players. GSS have the characteristic that the best-response of each player is weakly decreasing in the action of each of the other players.

There is a long literature developing the theory of GSC. Some of this work can be seen in Topkis (1978), Topkis (1979), Bulow, Geanakoplos, and Klemperer (1985), Lippman, Mamer, and McCardle (1987), Sobel (1988), Milgrom and Roberts (1990), Vives (1990), Milgrom and Shannon (1994), Milgrom and Roberts (1994), Zhou (1994), Shannon (1995), Villas-Boas (1997), Edlin and Shannon (1998), Echenique (2002), Echenique (2004), Quah (2007), and Quah and Strulovici (2009), among others. Extensive bibliographies are available in Topkis (1998), in Vives (1999), and in Vives (2005).

There is a growing literature on GSS: confer Amir (1996), Villas-Boas (1997), Amir and Lambson (2000), Schipper (2003), Zimper (2007), Roy and Sabarwal (2008), Acemoglu and Jensen (2009), Amir, Garcia, and Knauff (2010), Acemoglu and Jensen (2010), Roy and Sabarwal (2010), Jensen (2010), and Roy and Sabarwal (2012) among others.

This paper focuses on games with both strategic substitutes and strategic complements. Relatively little is known about such games even though several economic interactions exhibit these properties. For example, a classic application in Singh and Vives (1984) considers a duopoly in which one firm behaves as a Cournot-firm (exhibiting strategic substitutes) and the other as a Bertrand-firm (with strategic complements). Another example is that of a Becker (1968) type game of crime and 
punishment with a criminal and police: the criminal exhibits strategic substitutes (the greater is law enforcement, the lower is crime) and the police exhibit strategic complements (the greater is crime, the greater is law enforcement). Such games also arise in studies of pre-committment in industries with learning effects, see Tombak (2006), who terms these as games with strategic asymmetry. Moreover, Fudenberg and Tirole (1984) and Dixit (1987) also present examples of pre-committment where the strategic property of one player's action is opposite to that of the other player.

Games with both strategic substitutes and complements may behave differently from either GSC or GSS.

In a related paper, Monaco and Sabarwal (2011), we show that the nice order and structure properties of GSC do not survive a minimal introduction of strategic substitutes, in the following sense. Consider a game in which all-but-one players exhibit strategic complements (with one player exhibiting strict strategic complements), and the remaining player exhibits strict strategic substitutes. In this case, no two equilibria in the game are comparable (in the product order). Those results are stronger, and show that in any lattice game, if there is reason to believe that either (1) just one player has strict strategic complements and another player has strict strategic substitutes, or (2) just one player has strict strategic substitutes and has singleton-valued best-responses, then without any restrictions on the strategic interaction among the other players, no two equilibria are comparable.

In particular, our earlier result implies that in such cases, with multiple equilibria, there is no largest or smallest equilibrium, and therefore, the standard technique (in GSC) of using extremal equilibria to show monotone comparative statics in parameterized GSC is invalid.

In this paper, we first show that in parameterized games with both strategic substi- 
tutes and complements, equilibria do not decrease as the parameter increases. Moreover, using newer techniques, we provide conditions that guarantee that an increase in the parameter leads to an increase in the equilibrium; in other words, conditions under which monotone comparative statics is guaranteed.

For two-player games in which one player exhibits strategic substitutes, the other player exhibits strategic complements, and each player has a linearly ordered strategy space, we characterize monotone comparative statics via a condition on the best response of only the player with strategic substitutes. (No additional condition is imposed on the player with strategic complements.) The condition is intuitive and is based on a trade-off between the direct parameter effect and the indirect strategic substitute effect.

This characterization does not hold more generally: either for two-player games with more general strategy spaces, or for games with more players, as shown in several examples. In this regard, games with both strategic substitutes and complements behave differently from GSS.

For more general cases, we present sufficient conditions that guarantee monotone comparative statics. As in the two-player case, these conditions are needed only for players with strategic substitutes. The conditions are stronger than in the two-player case, but still involve a trade-off between the direct parameter effect and the indirect strategic substitute effect.

The paper is organized as follows. The next section gives several basic examples of games with strategic asymmetry. Section 3 presents the formal definition of such games. Section 4 shows that in such games there are no decreasing equilibria. Section 5 anaylzes two-player games and section 6 presents the results for multi-player games. Section 7 concludes. 


\section{Motivating Examples}

Example 1 (Differentiated Duopoly). Consider the differentiated duopoly in Singh and Vives (1984), where firm 1 chooses price as a strategic variable, and firm 2 chooses quantity. Inverse market demand for each firm is given by $p_{1}=a_{1}-b_{1} q_{1}-c q_{2}$ and $p_{2}=a_{2}-c q_{1}-b_{2} q_{2} 1$ Re-writing firm 1's demand yields $q_{1}\left(p_{1}, q_{2}\right)=\frac{1}{b_{1}}\left(a_{1}-c q_{2}-\right.$ $\left.p_{1}\right)$, and assuming zero cost, firm 1's profit is $\pi_{1}\left(p_{1}, q_{2}\right)=p_{1} q_{1}\left(p_{1}, q_{2}\right)$. Similarly, using firm 1's demand, and assuming zero cost, we may write firm 2's profit as $\pi_{2}\left(p_{1}, q_{2}\right)=$ $\left(a_{2}-\frac{c}{b_{1}}\left(a_{1}-c q_{2}-p_{1}\right)-b_{2} q_{2}\right) q_{2}$. It is easy to check that $\frac{\partial^{2} \pi_{1}}{\partial q_{2} \partial p_{1}}=-\frac{c}{b_{1}}<0$ and $\frac{\partial^{2} \pi_{2}}{\partial q_{2} \partial p_{1}}=\frac{c}{b_{1}}>0$. In other words, firm 1's best response is decreasing in firm 2's quantity choice, and firm 2's best response is increasing in firm 1's price choice. Indeed, (the linear) best responses are given as follows: for firm 1, $p_{1}=\frac{a_{1}-c q_{2}}{2}$, and for firm $2, q_{2}=\frac{a_{2} b_{1}-a_{1} c+c p_{1}}{2\left(b_{1} b_{2}-c^{2}\right)}$.

Example 2 (Crime and Punishment). Consider a simplified version of Becker (1968): there is a criminal (player 1) and a police force (player 2). When police enforce law with intensity $x \geq 0$, the benefit a criminal derives from commiting a crime of intensity $y \geq 0$ is $u_{C}(x, y)=\frac{\sqrt{y}}{2+x y}$. The benefit the police derive is $u_{P}(x, y)=-x c^{4}-\frac{y^{2}}{x}$, where $x c^{4}$ reflects cost of law enforcement and $\frac{y^{2}}{x}$ refers to damage due to criminal activity. The damage is increasing in criminal activity and decreasing in law enforcement. It is easy to check that for the police, $\frac{\partial^{2} u_{P}}{\partial x \partial y}=\frac{2 y}{x^{2}}>0$. The best response function for the police is given by $x=\frac{y}{c^{2}}$, and it is increasing in $y$. For the criminal, $\frac{\partial^{2} u_{C}}{\partial x \partial y}=\frac{\sqrt{y}}{2(2+x y)^{3}}(x y-6)$, and therefore, $\frac{\partial^{2} u_{C}}{\partial x \partial y}<0$, if, and only if, $x y<6$. Moreover, it is easy to compute that the criminal's payoff is concave in $y$, if, and only if, $x y<2+\sqrt{16 / 3} \approx 4.31$. With these constraints, the criminal's best response function is given by $y=\frac{2}{x}$ and it is decreasing in $x$. (For reference, it is easy

\footnotetext{
${ }^{1}$ As usual, we assume that $a_{1}, a_{2}, b_{1}, b_{2}, c>0$, and $b_{1} b_{2}-c^{2}>0$.
} 
to show that in Nash equilibrium, $x y=2$.)

Example 3 (Cournot Duopoly with Spillovers). Consider two firms, an incumbent (firm 1) and an entrant (firm 2) competing as Cournot duopolists, producing quantities $x_{1}$ and $x_{2}$, respectively. Inverse market demand is given by $p=$ $a-b\left(x_{1}+x_{2}\right)$. Firm 1's costs are linear, given by a constant marginal cost $c_{1}>0$. Thus, the incumbent's profit is given by $\pi_{1}\left(x_{1}, x_{2}\right)=\left(a-b\left(x_{1}+x_{2}\right)\right) x_{1}-c x_{1}$. As $\frac{\partial^{2} \pi_{1}}{\partial x_{2} \partial x_{1}}=-b<0$, firm 1's best response is decreasing in $x_{2}$. Indeed, firm 1's best response is given by $x_{1}=\frac{a-c_{1}-b x_{2}}{2 b}$. Suppose there is a one-way spillover from the incumbent to the entrant, say, in the form of defection of some employees with technologyspecific skill, and this lowers firm 1's costs. The spillover may depend on firm 1's output, and is denoted $s\left(x_{1}\right)$. Suppose firm 2's costs are given by $c_{2} x_{2} s\left(x_{1}\right)$. Its profits are given by $\pi_{2}\left(x_{1}, x_{2}\right)=\left(a-b\left(x_{1}+x_{2}\right)\right) x_{2}-c_{2} x_{2} s\left(x_{1}\right)$. In this case, $\frac{\partial^{2} \pi_{2}}{\partial x_{2} \partial x_{1}}=-b-c_{2} s^{\prime}\left(x_{1}\right)$,

and therefore, $\frac{\partial^{2} \pi_{2}}{\partial x_{2} \partial x_{1}}>0$ exactly when $s^{\prime}\left(x_{1}\right)<-\frac{b}{c_{2}}$. (Two spillover functions satisfying these conditions are: $s\left(x_{1}\right)=-\frac{2 b}{c_{2}} x_{1}$, and $s\left(x_{1}\right)=\ln \left(\frac{1}{x_{1}}\right)-\frac{b}{c_{2}} x_{1}$.) With this assumption on the spillover function, firm 2's best response is given by $x_{2}=\frac{a-c_{2} s\left(x_{1}\right)-b x_{1}}{2 b}$, and is increasing in $x_{1}$.

\section{Parameterized Lattice Games}

As usual, a lattice is a partially ordered set in which every two elements, $x$ and $y$, have a supremum, denoted $x \vee y$, and an infimum, denoted $x \wedge y$. A complete lattice is a lattice in which every non-empty subset has a supremum and infimum in the set.2 A function $f: X \rightarrow \mathbb{R}$ (where $X$ is a lattice) is quasi-supermodular if (1) $f(x) \geq f(x \wedge y) \Longrightarrow f(x \vee y) \geq f(y)$, and $(2) f(x)>f(x \wedge y) \Longrightarrow f(x \vee y)>f(y)$. A function $f: X \times T \rightarrow \mathbb{R}$ (where $X$ is a lattice and $T$ is a partially ordered set) satisfies

\footnotetext{
${ }^{2}$ This paper uses standard lattice terminology. See, for example, Topkis (1998).
} 
single-crossing property in $(x ; t)$ if for every $x^{\prime} \prec x^{\prime \prime}$ and $t^{\prime} \prec t^{\prime \prime},(1) f\left(x^{\prime}, t^{\prime}\right) \leq$ $f\left(x^{\prime \prime}, t^{\prime}\right) \Longrightarrow f\left(x^{\prime}, t^{\prime \prime}\right) \leq f\left(x^{\prime \prime}, t^{\prime \prime}\right)$, and $(2) f\left(x^{\prime}, t^{\prime}\right)<f\left(x^{\prime \prime}, t^{\prime}\right) \Longrightarrow f\left(x^{\prime}, t^{\prime \prime}\right)<f\left(x^{\prime \prime}, t^{\prime \prime}\right)$.

Consider finitely many players, $I$, and for each player $i$, a strategy space that is a partially ordered set, denoted $\left(X_{i}, \preceq_{i}\right)$, a real-valued payoff function, denoted $u_{i}\left(x_{i}, x_{-i}, t\right)$, and a partially ordered set of parameters, $T$. As usual, the product of the strategy spaces, denoted $(X, \preceq)$, is endowed with the product order and topology 3 The strategic game $\Gamma=\left\{\left(X_{i}, \preceq_{i}, u_{i}\right)_{i=1}^{I}, T\right\}$ is a parameterized lattice game if for every player $i$,

1. $X_{i}$ is a non-empty, complete lattice, and

2. For every every player $i$ and every $\left(x_{-i}, t\right), u_{i}$ is quasi-supermodular and upper semi-continuous in $x_{i}, 4$ and

3. For every player $i$ and every $x_{-i}, u_{i}$ satisfies single-crossing property in $\left(x_{i} ; t\right)$.

This general definition allows for games with strategic complements, games with strategic substitutes, and mixtures of the two.

For each $t \in T$, and for each player $i$, the best response of player $i$ to $x_{-i}$ is denoted $\beta_{t}^{i}\left(x_{-i}\right)$, and is given by $\arg \max _{x_{i} \in X_{i}} u_{i}\left(x_{i}, x_{-i}, t\right)$. As the payoff function is quasi-supermodular and upper semi-continuous, and the strategy space is compact in the order interval topology, for every $i$, and for every $\left(x_{-i}, t\right), \beta_{t}^{i}\left(x_{-i}\right)$ is a non-empty, complete sub-lattice. When convenient, we use $\bar{\beta}_{t}^{i}\left(x_{i}\right)=\sup \beta_{t}^{i}\left(x_{i}\right)$, and $\underline{\beta}_{t}^{i}\left(x_{i}\right)=$ $\inf \beta_{t}^{i}\left(x_{i}\right)$.

Moreover, single-crossing property in $\left(x_{i} ; t\right)$ implies that $\beta_{t}^{i}\left(x_{-i}\right)$ is non-decreasing in $t$ in the induced set order. (The standard induced set order is defined as follows:

\footnotetext{
${ }^{3}$ For notational convenience, we typically drop the index $i$ from the notation for the partial order.

${ }^{4}$ In the standard order interval topology.
} 
for non-empty subsets $A, B$ of a lattice $X, A \sqsubseteq_{i n} B$ in the induced set order, if for every $a \in A$, and for every $b \in B, a \wedge b \in A$, and $a \vee b \in B$.) Thus, every player $i$ 's best response satisfies: for every $t \preceq \hat{t}$ and for every $x_{-i}, \beta_{t}^{i}\left(x_{-i}\right) \sqsubseteq_{i n} \beta_{\hat{t}}^{i}\left(x_{-i}\right)$. Notice that when best response is singleton-valued, this is equivalent to the statement that the best response is a weakly increasing function in $t$ : for every $t \preceq \hat{t}$ and for every $x_{-i}, \beta_{t}^{i}\left(x_{-i}\right) \preceq \beta_{\hat{t}}^{i}\left(x_{-i}\right)$.

For each $t \in T$, let $\beta_{t}: X \rightarrow X$ given by $\beta_{t}(x)=\left(\beta_{t}^{i}\left(x_{-i}\right)\right)_{i \in I}$ denote the $\boldsymbol{j o i n t}$ best-response correspondence. From properties of player best responses, it follows that for every $t \preceq \hat{t}$ and for every $x, \beta_{t}(x) \sqsubseteq_{i n} \beta_{\hat{t}}(x)$. In other words, the joint best response is non-decreasing in $t$ in the induced set order. As earlier, if each player's best response is singleton-valued, then the joint best response is singleton-valused and weakly increasing in $t$ : for every $t \preceq \hat{t}$ and for every $x, \beta_{t}(x) \preceq \beta_{\hat{t}}(x)$.

As usual, for each $t \in T$, a (pure strategy) Nash equilibrium is a profile of player actions $x$ such that $x \in \beta_{t}(x)$. The equilibrium set at $t$ is given by $\mathcal{E}(t)=$ $\left\{x \in X \mid x \in \beta_{t}(x)\right\}$. Needless to say, at this level of generality, a lattice game may have no Nash equilibrium. For example, the textbook two-player matching pennies game is admissible here, and has no pure strategy Nash equilibrium. One may assume additional conditions to invoke standard results to guarantee existence of equilibrium via Brouwer-Schauder type theorems, or Kakutani-Glicksberg-Ky Fan type theorems, or other types of results. We do not make these assumptions so that our results apply whenever equilibrium exists, regardless of whether a specific equilibrium existence theorem is invoked, or whether an equilibrium is shown to exist directly in a game.

Of particular interest to us are cases where the best-response of a player is either increasing (the case of strategic complements) or decreasing (the case of strategic substitutes) with respect to the strategies of the other players. Here, increasing or decreasing are with respect to an appropriately defined set order, as follows. 
Recall that for each $t$, if the payoff function of player $i$ is quasi-supermodular in $x_{i}$, and satisfies the single-crossing property in $\left(x_{i} ; x_{-i}\right)$, then the best-response correspondence of player $i$ is nondecreasing in the induced set order. That is, for every $t$ and every $x_{-i}^{\prime} \preceq x_{-i}^{\prime \prime}, \beta_{t}^{i}\left(x_{-i}^{\prime}\right) \sqsubseteq_{i n} \beta_{t}^{i}\left(x_{-i}^{\prime \prime}\right)$. When player $i$ 's best response is a function, this translates into the standard definition of a weakly increasing function; for every $t$ and every $x_{-i}^{\prime} \preceq x_{-i}^{\prime \prime}, \beta_{t}^{i}\left(x_{-i}^{\prime}\right) \preceq \beta_{t}^{i}\left(x_{-i}^{\prime \prime}\right)$. Let us formalize this by saying that player $i$ has strategic complements, if for every $t$, player $i$ 's best response correspondence $\beta_{t}^{i}$ is non-decreasing in $x_{-i}$ in the induced set order.

Similarly, for each $t$, if the payoff function of player $i$ is quasi-supermodular in $x_{i}$, and satisfies the dual single-crossing property in $\left(x_{i} ; x_{-i}\right) \cdot 5$ then the best-response correspondence of player $i$ is nonincreasing in the induced set order. That is, for every $t$ and every $x_{-i}^{\prime} \preceq x_{-i}^{\prime \prime}, \beta_{t}^{i}\left(x_{-i}^{\prime \prime}\right) \sqsubseteq_{i n} \beta_{t}^{i}\left(x_{-i}^{\prime}\right)$. When player $i$ 's best response is a function, this translates into the standard definition of a weakly decreasing function: for every $t$ and every $x_{-i}^{\prime} \preceq x_{-i}^{\prime \prime}, \beta_{t}^{i}\left(x_{-i}^{\prime \prime}\right) \preceq \beta_{t}^{i}\left(x_{-i}^{\prime}\right)$. Let us formalize this by saying that player $i$ has strategic substitutes, if for every $t$, player $i$ 's best response correspondence $\beta_{t}^{i}$ is non-increasing in $x_{-i}$ in the induced set order.

Notice that the definitions of strategic complements and strategic substitutes are weak versions, because both admit a best response correspondence that is constant in other player actions. Therefore, it is useful to define strict versions of these ideas as well. Consider the following set order. For non-empty subsets $A, B$ of a lattice $X$, $A$ is strictly lower than $B$, denoted $A \sqsubset_{s} B$, if for every $a \in A$, and for every $b \in B, a \prec b$. This definition is a slight strengthening of the following set order

\footnotetext{
${ }^{5} \mathrm{~A}$ function $f: X \times T \rightarrow \mathbb{R}$ (where $X$ is a lattice and $T$ is a partially ordered set) satisfies $\boldsymbol{d u a l}$ single-crossing property in $(x ; t)$ if for every $x^{\prime} \prec x^{\prime \prime}$ and $t^{\prime} \prec t^{\prime \prime},(1) f\left(x^{\prime \prime}, t^{\prime}\right) \leq f\left(x^{\prime}, t^{\prime}\right) \Longrightarrow$ $f\left(x^{\prime \prime}, t^{\prime \prime}\right) \leq f\left(x^{\prime}, t^{\prime \prime}\right)$, and (2) $f\left(x^{\prime \prime}, t^{\prime}\right)<f\left(x^{\prime}, t^{\prime}\right) \Longrightarrow f\left(x^{\prime \prime}, t^{\prime \prime}\right)<f\left(x^{\prime}, t^{\prime \prime}\right)$. This is a natural generalization of Amir (1996).
} 
defined in Shannon (1995): $A$ is completely lower than $B$, denoted $A \sqsubseteq_{c} B$, if for every $a \in A$, and for every $b \in B, a \preceq b$. Notice that when $A$ and $B$ are non-empty, complete sub-lattices of $X$, (the case of best responses here,) $A$ is strictly lower than $B$, if, and only if, $\sup A \prec \inf B$; and similarly, $A$ is completely lower than $B$, if, and only if, $\sup A \preceq \inf B$.

Let us say that player $i$ has weakly strict strategic complements, if for every $t$, and every $x_{-i}^{\prime} \prec x_{-i}^{\prime \prime}, \beta_{t}^{i}\left(x_{-i}^{\prime}\right) \sqsubseteq_{c} \beta_{t}^{i}\left(x_{-i}^{\prime \prime}\right)$. In other words, for every $t$, player $i$ 's best response is increasing in $x_{-i}$ in the completely lower than set order. Notice that when best response is singleton-valued, weakly strict strategic complements is equivalent to strategic complements, and therefore, may not necessarily yield strictly increasing best responses. Say that player $i$ has strict strategic complements, if for every $t$, and every $x_{-i}^{\prime} \prec x_{-i}^{\prime \prime}, \beta_{t}^{i}\left(x_{-i}^{\prime}\right) \sqsubset_{s} \beta_{t}^{i}\left(x_{-i}^{\prime \prime}\right)$. In other words, for every $t$, player $i$ 's best response is increasing in $x_{-i}$ in the strictly lower than set order. Applying a result due to Shannon (1995), if for every $\left(x_{-i}, t\right)$, player $i$ 's payoff is strictly quasi-supermodular in $x_{i}, 6$ and for every $t$, player $i$ 's payoff satisfies strict singlecrossing property in $\left(x_{i}, x_{-i}\right)$, 7 then player $i$ has weakly strict strategic complements. Moreover, in finite-dimensional Euclidean spaces, Edlin and Shannon (1998) provide an additional intuitive and easy-to-use differentiable condition regarding strictly increasing marginal returns to derive a comparison in the strictly lower than set order, and therefore, to conclude that player $i$ has strict strategic complements.

Similarly, player $i$ has weakly strict strategic substitutes, if for every $t$, and every $x_{-i}^{\prime} \prec x_{-i}^{\prime \prime}, \beta_{t}^{i}\left(x_{-i}^{\prime \prime}\right) \sqsubseteq_{c} \beta_{t}^{i}\left(x_{-i}^{\prime}\right)$. In other words, for every $t$, player $i$ 's

\footnotetext{
${ }^{6} \mathrm{~A}$ function $f: X \rightarrow \mathbb{R}$ (where $X$ is a lattice) is strictly quasi-supermodular if for all unordered $x, y, f(x) \geq f(x \wedge y) \Longrightarrow f(x \vee y)>f(y)$.

${ }^{7} \mathrm{~A}$ function $f: X \times T \rightarrow \mathbb{R}$ (where $X$ is a lattice and $T$ is a partially ordered set) satisfies strict single-crossing property in $(x ; t)$ if for every $x^{\prime} \prec x^{\prime \prime}$ and $t^{\prime} \prec t^{\prime \prime}, f\left(x^{\prime}, t^{\prime}\right) \leq f\left(x^{\prime \prime}, t^{\prime}\right) \Longrightarrow$ $f\left(x^{\prime}, t^{\prime \prime}\right)<f\left(x^{\prime \prime}, t^{\prime \prime}\right)$.
} 
best response is decreasing in $x_{-i}$ in the completely lower than set order. Moreover, player $i$ has strict strategic substitutes, if for every $t$, and every $x_{-i}^{\prime} \prec x_{-i}^{\prime \prime}$, $\beta_{t}^{i}\left(x_{-i}^{\prime \prime}\right) \sqsubset_{s} \beta_{t}^{i}\left(x_{-i}^{\prime}\right)$. In other words, for every $t$, player $i$ 's best response is decreasing in $x_{-i}$ in the strictly lower than set order. The conditions for strict strategic complements and weakly strict strategic complements can be easily adapted for substitutes.

\section{Non-Decreasing Equilibrium Selections}

The following result shows that in a broad class of games, there are no decreasing selections of equilibria.

Theorem 1. In a parameterized lattice game, suppose one of the following conditions is satisfied.

1. One player has strict strategic substitutes and singleton-valued best response.

2. One player, say player $i$, has strict strategic substitutes and strict single-crossing property in $\left(x_{i} ; t\right)$.

Then for every $t^{*} \prec \hat{t}$, for every $x^{*} \in \mathcal{E}\left(t^{*}\right)$, and for every $\hat{x} \in \mathcal{E}(\hat{t}), \hat{x} \nprec x^{*}$.

Proof. Consider condition 1, and without loss of generality, suppose it is satisfied for player 1 . Suppose $\hat{x} \prec x^{*}$. As case 1 , consider $\hat{x}_{-1} \prec x_{-1}^{*}$. Then $x_{1}^{*}=\beta_{t^{*}}^{1}\left(x_{-1}^{*}\right) \prec$ $\beta_{t^{*}}^{1}\left(\hat{x}_{-1}\right) \preceq \beta_{\hat{t}}^{1}\left(\hat{x}_{-1}\right)=\hat{x}_{1}$, contradicting $\hat{x} \prec x^{*}$. Here, the strict inequality follows from strict strategic substitutes, and the weak inequality follows from single-crossing property in $\left(x_{1} ; t\right)$. As case 2 , consider $\hat{x}_{-1}=x_{-1}^{*}$ and $\hat{x}_{1} \prec x_{1}^{*}$. Then $x_{1}^{*}=\beta_{t^{*}}^{1}\left(x_{-1}^{*}\right)=$ $\beta_{t^{*}}^{1}\left(\hat{x}_{-1}\right) \preceq \beta_{\hat{t}}^{1}\left(\hat{x}_{-1}\right)=\hat{x}_{1}$, contradicting $\hat{x}_{1} \prec x_{1}^{*}$.

Consider condition 2, suppose it is satisfied for player 1 , and suppose $\hat{x} \prec x^{*}$. As case 1 , consider $\hat{x}_{-1} \prec x_{-1}^{*}$. Then $\beta_{t^{*}}^{1}\left(x_{-1}^{*}\right) \sqsubset_{s} \beta_{t^{*}}^{1}\left(\hat{x}_{-1}\right) \sqsubseteq_{c} \beta_{\hat{t}}^{1}\left(\hat{x}_{-1}\right)$, where the strictly 
lower than relation follows from strict strategic substitute, and the completely lower than relation follows from strict single-crossing property in $\left(x_{1} ; t\right)$. Consequently, $x_{1}^{*} \preceq \sup \beta_{t^{*}}^{1}\left(x_{-1}^{*}\right) \prec \inf \beta_{t^{*}}^{1}\left(\hat{x}_{-1}\right) \preceq \inf \beta_{\hat{t}}^{1}\left(\hat{x}_{-1}\right) \preceq \hat{x}_{1}$, contradicting $\hat{x}_{1} \prec x_{1}^{*}$. As case 2 , consider $\hat{x}_{-1}=x_{-1}^{*}$ and $\hat{x}_{1} \prec x_{1}^{*}$. Then $\beta_{t^{*}}^{1}\left(x_{-1}^{*}\right)=\beta_{t^{*}}^{1}\left(\hat{x}_{-1}\right) \sqsubseteq_{c} \beta_{\hat{t}}^{1}\left(\hat{x}_{-1}\right)$, whence $x_{1}^{*} \preceq \sup \beta_{t^{*}}^{1}\left(x_{-1}^{*}\right)=\sup \beta_{t^{*}}^{1}\left(\hat{x}_{-1}\right) \preceq \inf \beta_{\hat{t}}^{1}\left(\hat{x}_{-1}\right) \preceq \hat{x}_{1}$, contradicting $\hat{x}_{1} \prec x_{1}^{*}$.

This theorem presents conditions on one player only to derive equilibrium selection results. In particular, if one player exhibits strict strategic substitutes and has a singleton-valued best response, then without any restrictions on the strategic interdependence among the other players, there are no decreasing selections of equilibria. In particular, this theorem does not require other players to exhibit either strategic substitutes or strategic complements.

Example 3 (Cournot Duopoly with Spillovers), continued. Recall the best responses in the Cournot duopoly with spillovers example above, given by $\beta^{1}\left(x_{2}\right)=$ $\max \left\{\frac{a-b x_{2}-c_{1}}{2 b}, 0\right\}$, and $\beta^{2}\left(x_{1}\right)=\max \left\{\frac{a-b x_{1}-c_{2} s\left(x_{1}\right)}{2 b}, 0\right\}$. Suppose the spillover function is given by $s\left(x_{1}\right)=\frac{2}{3} x_{1}^{3}-x_{1}^{2}-\frac{x_{1}}{2}+3$. This game satisfies condition 1 in the previous theorem 8 Given parameter values $a=15, b=\frac{1}{2}, c_{1}=11, c_{2}=3$, it is easy to check that there are three Nash equilibria: $x^{*}=(2,4), y^{*}=\left(\frac{1}{2}, 7\right)$, and $z^{*}=(4,0)$, shown in figure 1. If we increase parameter $a$ to 17 , both best responses increase, and there are 3 new equilibria: $\hat{x}^{*}=(1.686,8.627), \hat{y}^{*}=(1,10)$, and $\hat{z}^{*}=(6,0)$. Notice that no new equilibrium is lower than any old equilibrium, as predicted by theorem 1. Moreover, firm 1's output in $\hat{x}^{*}=(1.686,8.627)$ is lower than its output in $x^{*}=(2,4)$, showing that in general, we cannot strengthen the conclusion of this theorem to conclude increasing equilibria.

Theorem 1 shows that in the presence of strategic substitutes, there are no decreasing equilibrium selections. Moreover, the example shows that in general, this

\footnotetext{
${ }^{8}$ It satisfies condition 2 as well, when considering $a$ as the parameter.
} 


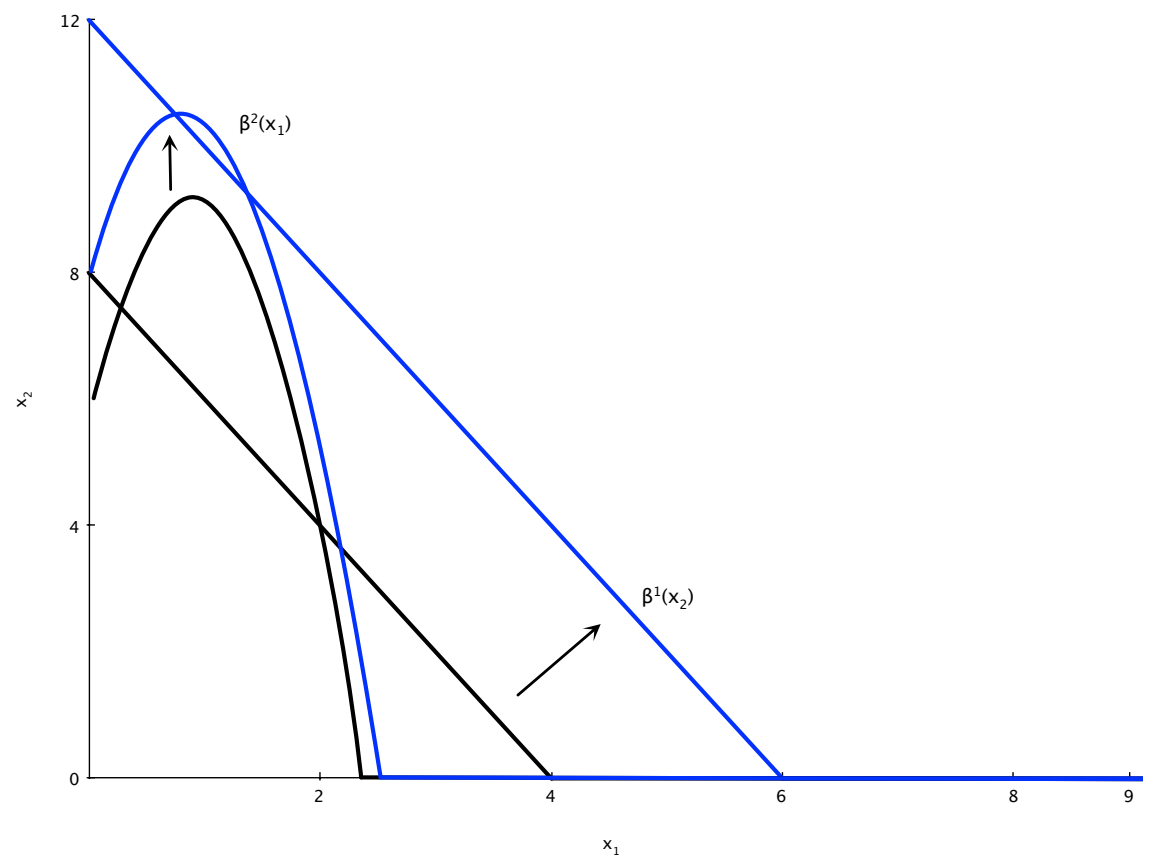

Figure 1: Non-Decreasing Equilibria For Cournot Duopoly With Spillovers

result cannot be strengthened to conclude monotone comparative statics. That is, when a parameter increases, it is possible that there are some higher equilibria, but it is also possible that there are equilbria that are not higher. As a step toward monotone comparative statics, the next section characterizes increasing equilibria for two-player games.

\section{Two-Player Parameterized Lattice Games}

This section considers two-player games in which one player exhibits strategic substitutes and the other one exhibits strategic comlements. For convenience, let's term such games two-player games with strategic asymmetry. In this setting, we present results characterizing monotone comparative statics. 
Theorem 2. Consider a two-player, parameterized lattice game, in which player 1 has strategic substitutes, and player 2 has strategic complements. Suppose strategy spaces are linearly ordered and best responses are singleton-valued.

For every $t^{*} \preceq \hat{t}$, for every $x^{*} \in \mathcal{E}\left(t^{*}\right)$, and for every $\hat{x} \in \mathcal{E}(\hat{t})$,

$$
\begin{aligned}
& \text { 1. } x_{2}^{*} \preceq \hat{x}_{2} \\
& \text { 2. } x^{*} \preceq \hat{x} \Longleftrightarrow x_{1}^{*} \preceq \beta_{\hat{t}}^{1} \circ \beta_{\hat{t}}^{2}\left(x_{1}^{*}\right)
\end{aligned}
$$

Proof. Consider statement 1. As case 1 , suppose $x_{1}^{*} \preceq \hat{x}_{1}$. Then $x_{2}^{*}=\beta_{t^{*}}^{2}\left(x_{1}^{*}\right) \preceq$ $\beta_{t^{*}}^{2}\left(\hat{x}_{1}\right) \preceq \beta_{\hat{t}}^{2}\left(\hat{x}_{1}\right)=\hat{x}_{2}$, where the first inequality follows from strategic complements, and the second from single-crossing property. As case 2, consider $x_{1}^{*} \npreceq \hat{x}_{1}$. Linearly ordered strategies implies $\hat{x}_{1} \prec x_{1}^{*}$. In this case, $\hat{x}_{2} \nprec x_{2}^{*}$. (For if $\hat{x}_{2} \prec x_{2}^{*}$, then $x_{1}^{*}=\beta_{t^{*}}^{1}\left(x_{2}^{*}\right) \preceq \beta_{\hat{t}}^{1}\left(x_{2}^{*}\right) \preceq \beta_{\hat{t}}^{1}\left(\hat{x}_{2}\right)=\hat{x}_{1}$, where, the first inequality follows from singlecrossing property and the second from strategic substitutes. This contradicts $\hat{x}_{1} \prec$ $x_{1}^{*}$.) Linearly ordered strategies now yields $x_{2}^{*} \preceq \hat{x}_{2}$.

Consider statement 2. For sufficiency, suppose $x^{*} \preceq \hat{x}$. Then $x_{1}^{*} \preceq \hat{x}_{1}$, and strategic complements implies $\beta_{\hat{t}}^{2}\left(x_{1}^{*}\right) \preceq \beta_{\hat{t}}^{2}\left(\hat{x}_{1}\right)$, whence $x_{1}^{*} \preceq \hat{x}_{1}=\beta_{\hat{t}}^{1} \beta_{\hat{t}}^{2}\left(\hat{x}_{1}\right) \preceq \beta_{\hat{t}}^{1} \beta_{\hat{t}}^{2}\left(x_{1}^{*}\right)$, where the inequality follows from strategic substitutes. For necessity, suppose $x^{*} \npreceq$ $\hat{x}$. Then, using statement $1, x_{1}^{*} \npreceq \hat{x}_{1}$, and linear order implies $\hat{x}_{1} \prec x_{1}^{*}$. Thus, $\hat{x}_{2}=\beta_{\hat{t}}^{2}\left(\hat{x}_{1}\right) \preceq \beta_{\hat{t}}^{2}\left(x_{1}^{*}\right)$, where the inequality follows from strategic complements. Now, using strategic substitutes yields $\beta_{\hat{t}}^{1} \beta_{\hat{t}}^{2}\left(x_{1}^{*}\right) \preceq \beta_{\hat{t}}^{1}\left(\hat{x}_{2}\right)=\hat{x}_{1} \prec x_{1}^{*}$, as desired.

This result formalizes the intuition that in a two-player game with strategic asymmetry, when the parameter (weakly) increases, the equilibrium response of the player with strategic complements is always (weakly) higher. For monotone comparative statics, we also need the equilibrium response of the player with strategic subsitutes to be (weakly) higher; this is characterized by the second condition. That is, $x_{1}^{*} \preceq \hat{x}_{1}$ is equivalent to $x_{1}^{*} \preceq \beta_{\hat{t}}^{1} \circ \beta_{\hat{t}}^{2}\left(x_{1}^{*}\right)$. 
The condition $x_{1}^{*} \preceq \beta_{\hat{t}}^{1} \circ \beta_{\hat{t}}^{2}\left(x_{1}^{*}\right)$ can be viewed as follows. Starting from an existing equilibrium strategy for player $1, x_{1}^{*}$ at $t=t^{*}$, an increase in $t$ has two effects on $\beta_{(\cdot)}^{1}(\cdot)$. One effect is an increase in $\beta^{1}$, because best-response is nondecreasing in $t$. (This is the direct effect of an increase in $t$, arising from the single-crossing property in $\left(x_{1} ; t\right)$.) The other effect is a decrease in $\beta^{1}$, because an increase in $t$ increases $\beta_{t}^{2}\left(x_{1}^{*}\right)$, and $\beta_{1}$ is decreasing in $x_{2}$, due to strategic substitutes. (This is the indirect effect arising from player 1's response to player 2's response to an increase in $t$.) The condition says that for player 1 , as long as the indirect strategic substitute effect does not dominate the direct parameter effect, the new equilibrium response of player 1 is (weakly) larger than $x_{1}^{*}$. This can be viewed explicitly in the following example.

Example 1 (Differentiated Duopoly), continued. Consider the differentiated duopoly described above, parameterized by $t=a_{1}=a_{2}$, and recall that best responses are given as follows: for firm $1, \beta_{t}^{1}\left(q_{2}\right)=\frac{t-c q_{2}}{2}$, and for firm $2, \beta_{t}^{2}\left(p_{1}\right)=\frac{t b_{1}-t c+c p_{1}}{2\left(b_{1} b_{2}-c^{2}\right)}$. With the same assumptions as in Singh and Vives (1984), (that is, $b_{1}-c>0$ and $b_{1} b_{2}-c^{2}>0$,) best response of both players is increasing in $t$. Suppose $t=2, b_{1}=b_{2}=$ 2 , and $c=1$. In this case, the unique Nash equilibrium is given by $\left(p_{1}^{*}, q_{2}^{*}\right)=\left(\frac{2}{3}, \frac{2}{5}\right)$. Consider an increase to $\hat{t}=3$. In this case, $\beta_{\hat{t}}^{1}\left(\beta_{\hat{t}}^{2}\left(p_{1}^{*}\right)\right)=\frac{43}{36}>\frac{2}{3}=p_{1}^{*}$, and therefore, the new equilibrium is higher than the old equilibrium, as shown in figure 2. Indeed, the new equilbrium is $\left(\hat{p}_{1}, \hat{q}_{2}\right) \approx(1.15,0.69)$. (For reference, profits of both firms have gone up as well, from $(0.31,0.29)$ to $(0.39,0.72)$.)

For completeness, figure 3 represents graphically the case where the direct effect does not dominate the indirect effect. If best responses change in the manner shown in figure 3 , the composite effect is lower, $\left(\beta_{\hat{t}}^{1}\left(\beta_{\hat{t}}^{2}\left(p_{1}^{*}\right)\right)<p_{1}^{*},\right)$ and as theorem 2 predicts, the new equilbrium is not higher than the old equilibrium.

In order to characterize increasing equilibria with best response correspondences, consider the following lemmas. 


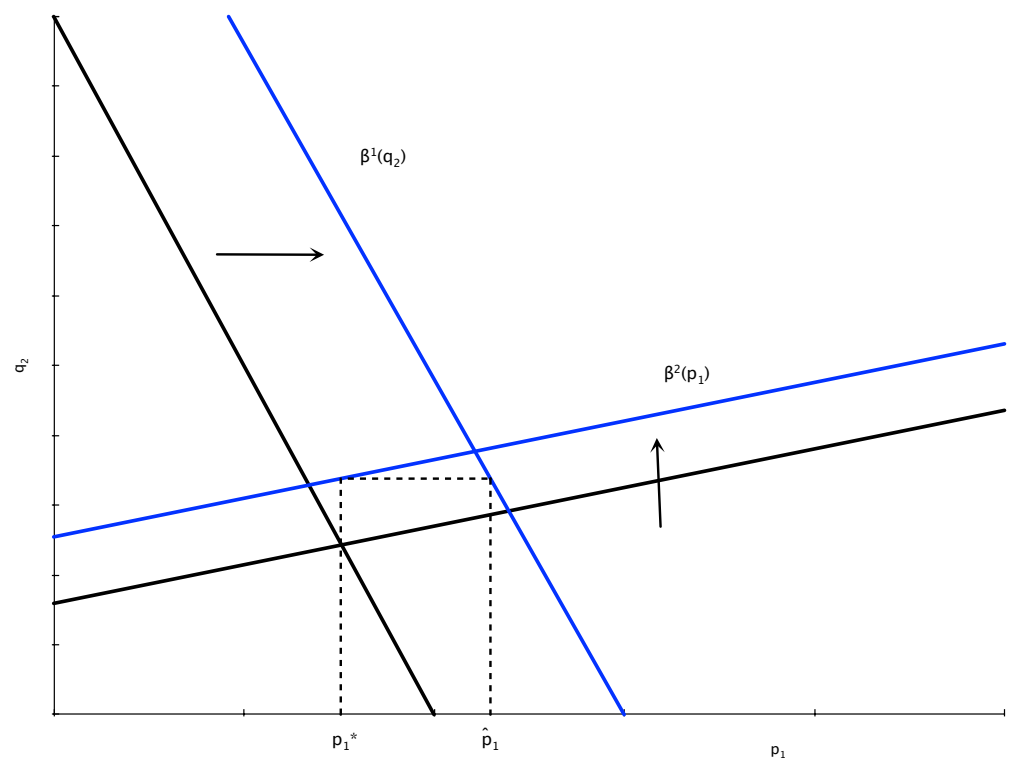

Figure 2: Increasing Equilibria: Differentiated Duopoly

Lemma 1. Consider a two-player, parameterized lattice game, in which player 1 has strict strategic substitutes, and player 2 has weakly strict strategic complements. Suppose strategy spaces are linearly ordered. For every $t^{*} \preceq \hat{t}$, for every $x^{*} \in \mathcal{E}\left(t^{*}\right)$, and for every $\hat{x} \in \mathcal{E}(\hat{t}), x_{2}^{*} \preceq \hat{x}_{2}$.

Proof. Suppose $x_{2}^{*} \npreceq \hat{x}_{2}$. Then linear order implies $\hat{x}_{2} \prec x_{2}^{*}$. Thus $\beta_{t^{*}}^{1}\left(x_{2}^{*}\right) \sqsubseteq_{\text {in }}$ $\beta_{\hat{t}}^{1}\left(x_{2}^{*}\right) \sqsubset_{s} \beta_{\hat{t}}^{1}\left(\hat{x}_{2}\right)$, where the induced set order inequality follows from single-crossing property in $\left(x_{1} ; t\right)$, and the strict set order inequality follows from strict strategic substitutes. This implies $x_{1}^{*} \preceq \bar{\beta}_{t^{*}}^{1}\left(x_{2}^{*}\right) \preceq \bar{\beta}_{\hat{t}}^{1}\left(x_{2}^{*}\right) \prec \underline{\beta}_{\hat{t}}^{1}\left(\hat{x}_{2}\right) \preceq \hat{x}_{1}$. Therefore, $\beta_{t^{*}}^{2}\left(x_{1}^{*}\right) \sqsubseteq_{\text {in }}$ $\beta_{\hat{t}}^{2}\left(x_{1}^{*}\right) \sqsubseteq_{c} \beta_{\hat{t}}^{2}\left(\hat{x}_{1}\right)$, where the induced set order inequality follows from single-crossing property in $\left(x_{2} ; t\right)$, and the completely lower set order inequality follows from weakly strict strategic complements. This implies $x_{2}^{*} \preceq \bar{\beta}_{t^{*}}^{2}\left(x_{1}^{*}\right) \preceq \bar{\beta}_{\hat{t}}^{2}\left(x_{1}^{*}\right) \preceq \underline{\beta}_{\hat{t}}^{2}\left(\hat{x}_{1}\right) \preceq \hat{x}_{2}$, a contradiction.

Lemma 2. Consider a two-player, parameterized lattice game, in which player 1 has 


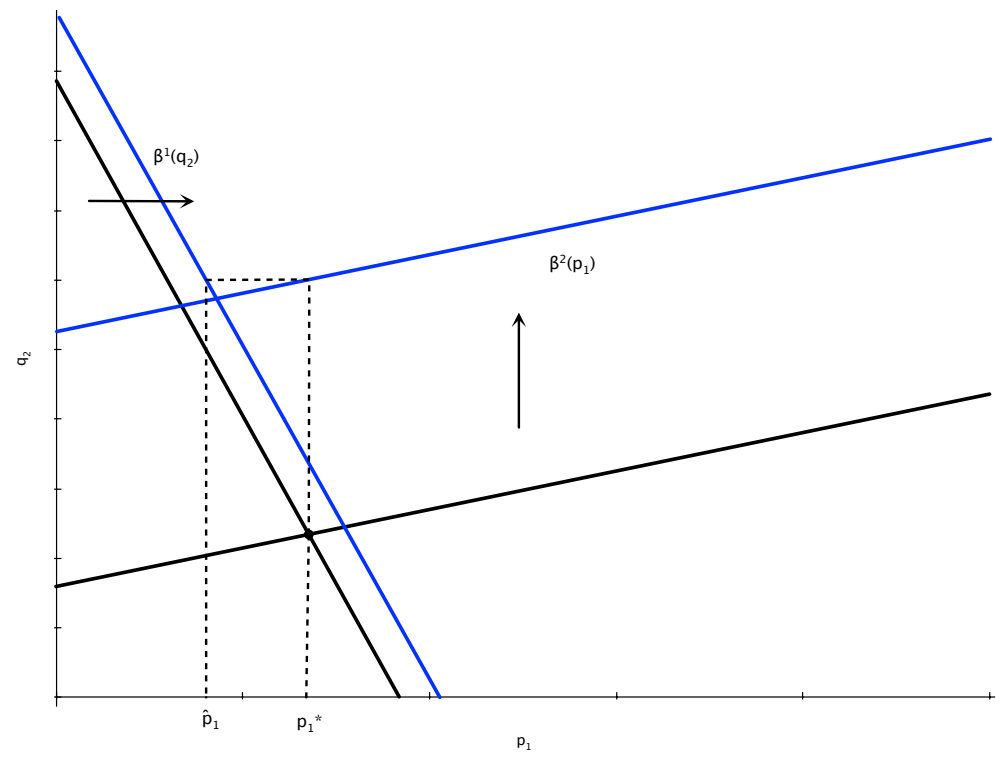

Figure 3: Non-increasing Equilibria: Differentiated Duopoly

strategic substitutes, and player 2 has strategic complements. Suppose strategy spaces are linearly ordered. For every $t^{*} \preceq \hat{t}$, for every $x^{*} \in \mathcal{E}\left(t^{*}\right)$, and for every $\hat{x} \in \mathcal{E}(\hat{t})$, $x_{1}^{*} \preceq \hat{x}_{1} \Longrightarrow x_{1}^{*} \preceq \bar{\beta}_{\hat{t}}^{1} \underline{\beta}_{\hat{t}}^{2}\left(x_{1}^{*}\right)$.

Proof. $x_{1}^{*} \preceq \hat{x}_{1}$ implies $\underline{\beta}_{\hat{t}}^{2}\left(x_{1}^{*}\right) \preceq \underline{\beta}_{\hat{t}}^{2}\left(\hat{x}_{1}\right) \preceq \hat{x}_{2}$, where the first inequality follows from strategic complements. This, in turn, implies, $x_{1}^{*} \preceq \hat{x}_{1} \preceq \bar{\beta}_{\hat{t}}^{1}\left(\hat{x}_{2}\right) \preceq \bar{\beta}_{\hat{t}}^{1} \beta_{\hat{t}}^{2}\left(x_{1}^{*}\right)$, where the last inequality follows from strategic substitutes.

Lemma 3. Consider a two-player, parameterized lattice game, in which player 1 has weakly strict strategic substitutes, and player 2 has strict strategic complements. Suppose strategy spaces are linearly ordered. For every $t^{*} \preceq \hat{t}$, for every $x^{*} \in \mathcal{E}\left(t^{*}\right)$, and for every $\hat{x} \in \mathcal{E}(\hat{t}), \hat{x}_{1} \prec x_{1}^{*} \Longrightarrow \bar{\beta}_{\hat{t}}^{1} \underline{\beta}_{\hat{t}}^{2}\left(x_{1}^{*}\right) \prec x_{1}^{*}$.

Proof. Using strict strategic complements, $\hat{x}_{1} \prec x_{1}^{*}$ implies $\beta_{\hat{t}}^{2}\left(\hat{x}_{1}\right) \sqsubset_{s} \beta_{\hat{t}}^{2}\left(x_{1}^{*}\right)$, and therefore, $\hat{x}_{2} \preceq \bar{\beta}_{\hat{t}}^{2}\left(\hat{x}_{1}\right) \prec \underline{\beta}_{\hat{t}}^{2}\left(x_{1}^{*}\right)$. Using weakly strict strategic substitutes, it follows that $\beta_{\hat{t}}^{1}\left(\underline{\beta}_{\hat{t}}^{2}\left(x_{1}^{*}\right) \sqsubseteq_{c} \beta_{\hat{t}}^{1}\left(\hat{x}_{2}\right)\right.$. Consequently, $\bar{\beta}_{\hat{t}}^{1} \underline{\beta}_{\hat{t}}^{2}\left(x_{1}^{*}\right) \preceq \underline{\beta}_{\hat{t}}^{1}\left(\hat{x}_{2}\right) \preceq \hat{x}_{1} \prec x_{1}^{*}$. 
These lemmas yield the following theorem immediately.

Theorem 3. Consider a two-player, parameterized lattice game, in which player 1 has strict strategic substitutes, and player 2 has strict strategic complements. Suppose strategy spaces are linearly ordered.

For every $t^{*} \preceq \hat{t}$, for every $x^{*} \in \mathcal{E}\left(t^{*}\right)$, and for every $\hat{x} \in \mathcal{E}(\hat{t})$,

1. $x_{2}^{*} \preceq \hat{x}_{2}$

2. $x^{*} \preceq \hat{x} \Longleftrightarrow x_{1}^{*} \preceq \bar{\beta}_{\hat{t}}^{1} \circ \underline{\beta}_{\hat{t}}^{2}\left(x_{1}^{*}\right)$

These results are valid for two-player games of strategic asymmetry with linearly ordered strategy spaces. To investigate more general cases, notice first that these results may not necessarily hold with more general strategy spaces, as shown in the next example.

Example 4 (Crime and Punishment-2). Consider another version of crime and punishment: there is a criminal (player 1) and a police force (player 2). The criminal has four actions: no crime $\left(a_{1}\right)$, grand theft auto $\left(a_{2}\right)$, bank robbery $\left(a_{3}\right)$, and both grand theft auto and bank robbery $\left(a_{4}\right)$, with $a_{1} \prec a_{2} \prec a_{4}, a_{1} \prec a_{3} \prec a_{4}$, and $a_{2}$ and $a_{3}$ are unordered. This makes $X_{1}=\left\{a_{1}, a_{2}, a_{3}, a_{4}\right\}$ into a lattice that is not linearly ordered. The police have two actions: low enforcement $\left(b_{1}\right)$ and high enforcement $\left(b_{2}\right)$, with $b_{1} \prec b_{2}$. Suppose payoffs are given in the left panel of figure 4 .

It is easy to check that the criminal exhibits strategic substitutes and the police exhibit strategic complements. This game has a unique Nash equilibrium: $\left(a_{2}, b_{1}\right)$.

Now suppose the bank receives a new, large, cash deposit - the equivalent of an increase in a parameter representing potential value of the bank's deposits. Denote the new parameter $\hat{t}$. The new game is given in the right panel of figure 4 . It is easy to check that compared to the left panel, the best response for each player is non- 

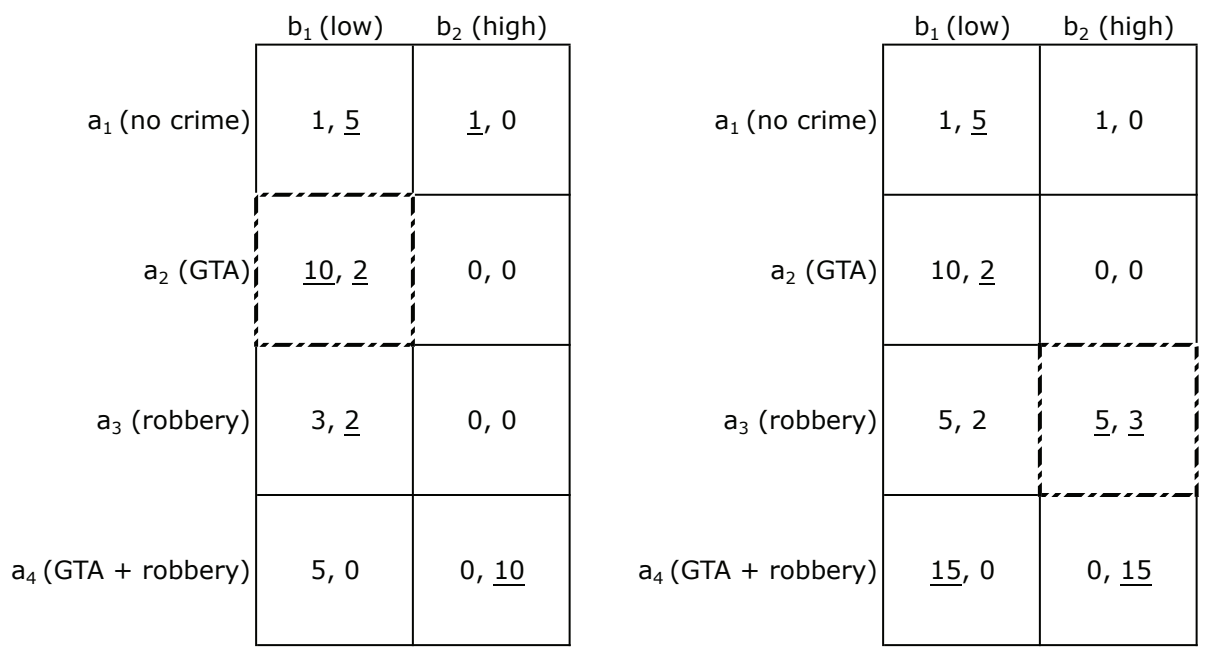

Figure 4: Crime and Punishment-2

decreasing; that is, the single-crossing property is satisfied. The new unique Nash equilibrium is $\left(a_{3}, b_{2}\right)$, which is not comparable to $\left(a_{2}, b_{1}\right)$, because $a_{2}$ and $a_{3}$ are not comparable.

Notice that the conditions of theorem 2 are satisfied in this case, because $a_{2} \prec$ $a_{4}=\beta_{\hat{t}}^{1}\left(\beta_{\hat{t}}^{2}\left(a_{2}\right)\right)$. This example shows that when we try to extend the analysis to non-linearly ordered lattices, theorem 2 does not necessarily hold.

To investigate generalization in another direction, let's consider cases with more than two players.

\section{Multi-Player Parameterized Lattice Games}

As the following example shows, when there are more than two players, even with linearly ordered strategy spaces, an analogue of theorem 2 may not necessarily hold. Example 4 (Crime and Punishment-2), continued. Consider another version 
of crime and punishment: there is a criminal (player 1) and two police forces (players 2 and 3 ). The criminal has four actions: $a_{1}, a_{2}, a_{3}$, and $a_{4}$, with the range of criminal activity increasing in intensity $a_{1} \prec a_{2} \prec a_{3} \prec a_{4}$. Police force 1 has two actions: low enforcement $\left(b_{1}\right)$ and high enforcement $\left(b_{2}\right)$, with $b_{1} \prec b_{2}$. Police force 2 has two actions: low enforcement $\left(c_{1}\right)$ and high enforcement $\left(c_{2}\right)$, with $c_{1} \prec c_{2}$. Suppose payoffs are given in figure 5 .

It is easy to check that the criminal exhibits strategic substitutes and both police forces exhibit strategic complements. The unique Nash equilibrium is given by $x^{*}=$ $\left(a_{3}, b_{1}, c_{1}\right)$.
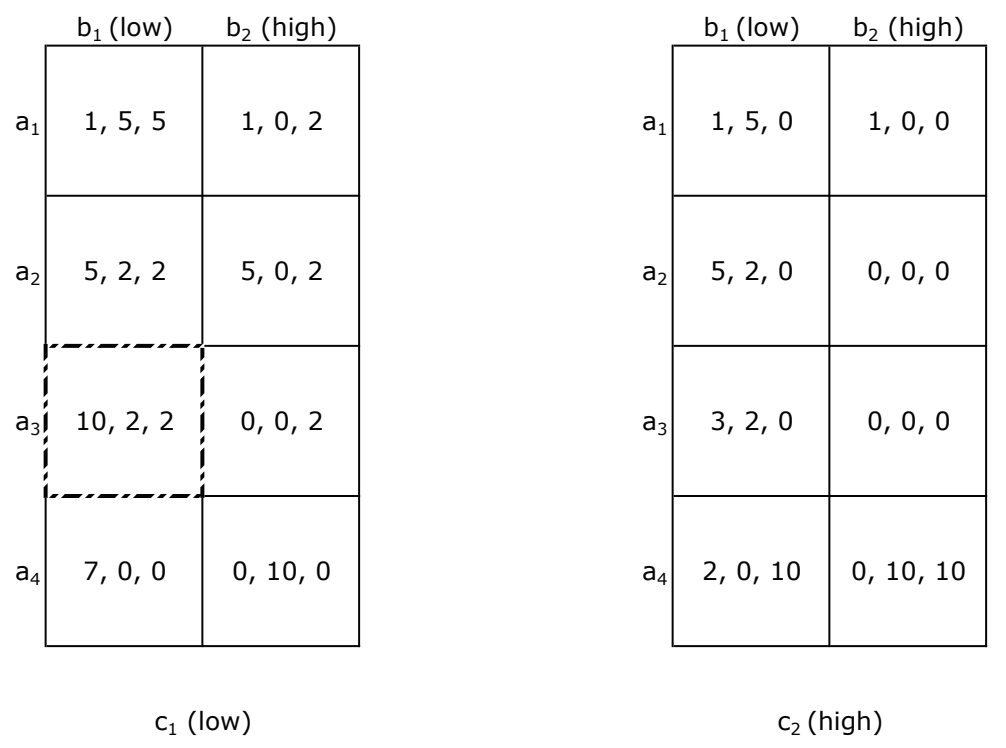

Figure 5: Crime and Punishment-2, before parameter change

Suppose, as earlier, an increase in the parameter corresponds to an increase in the value of criminal activity to the criminal. Denote the new parameter $\hat{t}$, and suppose the new payoffs are given in figure 6. The unique Nash equilibrium is given by $\hat{x}=\left(a_{2}, b_{2}, c_{2}\right)$, and this is not comparable to $x^{*}=\left(a_{3}, b_{1}, c_{1}\right)$.

Notice that the second iterate condition from theorem 2 would be as follows: $x_{1}^{*} \preceq$ 


\begin{tabular}{|c|c|c|}
\hline & $b_{1}$ (low) & $b_{2}$ (high) \\
\hline$a_{1}$ & $1,5,5$ & $1,0,2$ \\
\hline$a_{2}$ & $5,2,2$ & $5,0,2$ \\
\hline$a_{3}$ & $10,2,2$ & $10,0,2$ \\
\hline$a_{4}$ & $15,0,0$ & $7,10,0$ \\
\hline
\end{tabular}

$\mathrm{c}_{1}$ (low)

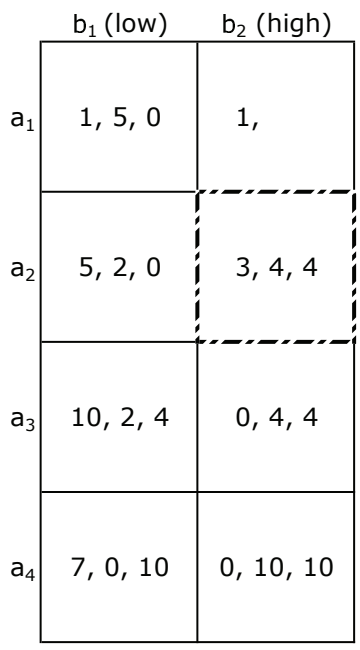

$\mathrm{C}_{2}$ (high)

Figure 6: Crime and Punishment-2, after parameter change

$\beta_{\hat{t}}^{1}\left(\beta_{\hat{t}}^{2}\left(x_{-2}^{*}\right), \beta_{\hat{t}}^{3}\left(x_{-3}^{*}\right)\right)$. This condition is satisfied, because $\beta_{\hat{t}}^{2}\left(a_{3}, c_{1}\right)=b_{1}, \beta_{\hat{t}}^{3}\left(a_{3}, b_{1}\right)=$ $c_{2}$, and therefore, $\hat{x}_{1}^{*}=a_{3} \preceq a_{3}=\beta_{\hat{t}}^{1}\left(b_{1}, c_{2}\right)=\beta_{\hat{t}}^{1}\left(\beta_{\hat{t}}^{2}\left(x_{-2}^{*}\right), \beta_{\hat{t}}^{3}\left(x_{-3}^{*}\right)\right)$.

These examples show that a straightforward application of theorem 2 may not necessarily work for more general cases 9 We now develop results that can be applied to more general cases.

The strategic game $\Gamma=\left\{\left(X_{i}, \preceq_{i}, u_{i}\right)_{i=1}^{I}, T\right\}$ is a parameterized lattice game, if for every player $i$,

1. The strategy space of player $i$ is $X_{i}$, a non-empty, compact, convex, sub-lattice of $\mathcal{X}^{i}$, where $\mathcal{X}^{i}$ is a locally convex and Hausdorff lattice vector space in the order interval topology 10 Let $\bar{x}_{i}=\sup X_{i}$.

\footnotetext{
${ }^{9}$ An additional counter-example can be constructed where two players exhibit strategic substitutes and one player exhibits strategic complements.

${ }^{10} \mathrm{~A}$ lattice vector space is a (real) vector space that is a lattice as well.
} 
2. $X=X^{1} \times \cdots \times X^{I}$ is the overall strategy space with the product order and topology, and $T$ is a partially ordered set.

3. For every player $i, u_{i}: X \times T \rightarrow \mathbb{R}$ is continuous in $x$, quasi-supermodular and quasi-concave in $x_{i}$ and satisfies single-crossing property in $\left(x_{i} ; t\right)$.

Theorem 4. Consider a parameterized lattice game in which players $1, \ldots, J$ have strategic substitutes and $J+1, \ldots$, I have strategic complements. Suppose best-responses are singleton-valued.

For every $t^{*} \preceq \hat{t}$ and every $x^{*} \in \mathcal{E}\left(t^{*}\right)$, let $\hat{y}=\left(\hat{y}_{i}\right)_{i \in I}$ be defined as follows: $\hat{y}_{i}=$ $\beta_{\hat{t}}^{i}\left(x_{-i}^{*}\right)$, for $i=1, \ldots, J$, and $\hat{y}_{i}=\beta_{\hat{t}}^{i}\left(\left(\hat{y}_{j}\right)_{j=1}^{J} ;\left(\bar{x}_{j}\right)_{j=J+1, j \neq i}^{I}\right)$, for $i=J+1, \ldots, I$. If for $i=1, \ldots, J, x_{i}^{*} \preceq \beta_{\hat{t}}^{i}\left(\hat{y}_{-i}\right)$, then there is $\hat{x} \in \mathcal{E}(\hat{t})$ such that $x^{*} \preceq \hat{x}$.

Proof. For $i=1, \ldots, I$, let $B_{i}=\left[x_{i}^{*}, \hat{y}_{i}\right]$, and let $B=\times_{i=1}^{I} B_{i}$. For $i=1, \ldots, I$, consider $\beta_{\hat{t}}^{i}$ on $B_{-i}$. Notice that $x_{i}^{*} \preceq \beta_{\hat{t}}^{i}\left(\hat{y}_{-i}\right)$ by assumption, and $\beta_{\hat{t}}^{i}\left(x_{-i}^{*}\right)=\hat{y}_{i}$, by definition. Therefore, $\beta_{\hat{t}}^{i}\left(B_{-i}\right) \subseteq B_{i}$; that is, $\beta_{\hat{t}}^{i}$ restricted to $B_{-i}$ maps into $B_{i}$. Similarly, for $i=J+1, \ldots, I$, consider $\beta_{\hat{t}}^{i}$ on $B_{-i}$. Single-crossing property in $\left(x_{i} ; t\right)$ yields $x_{i}^{*} \preceq \beta_{\hat{t}}^{i}\left(x_{-i}^{*}\right)$ and also, $\beta_{\hat{t}}^{i}\left(\hat{y}_{-i}\right)=\beta_{\hat{t}}^{i}\left(\left(\hat{y}_{j=1}^{J}\right) ;\left(\hat{y}_{j}\right)_{j=J+1, j \neq i}^{I} \preceq \beta_{\hat{t}}^{i}\left(\left(\hat{y}_{j=1}^{J}\right) ;\left(\bar{x}_{j}\right)_{j=J+1, j \neq i}^{I}\right)=\right.$ $\hat{y}_{i}$, where the inequality follows from $\left(\hat{y}_{j}\right)_{j=J+1, j \neq i}^{I} \preceq\left(\sup X^{j}\right)_{j=J+1, j \neq i}^{I}=\left(\bar{x}_{j}\right)_{j=J+1, j \neq i}^{I}$. Therefore, $\beta_{\hat{t}}^{i}\left(B_{-i}\right) \subseteq B_{i}$. Consequently, the joint best response function satisfies $\beta_{\hat{t}}(B) \subseteq B$; that is, the restriction of $\beta$ to $B$ is a self-map, and applying BrouwerSchauder-Tychonoff's theorem, there is a fixed point $\hat{x} \in \mathcal{E}(\hat{t})$ such that $x^{*} \preceq \hat{x}$.

The condition for multi-player games in theorem 4 is stronger than the condition characterizing increasing equilibria in two-player games (in theorem 2). This can be seen as follows. Consider a two-player game in which player 1 has strategic substitutes and player 2 has strategic complements. Notice that by the single-crossing property in $\left(x_{1} ; t\right), x_{1}^{*} \preceq \beta_{\hat{t}}^{1}\left(x_{2}^{*}\right)=\hat{y}_{1}$, and therefore, using $\hat{y}_{2}=\beta_{\hat{t}}^{2}\left(\hat{y}_{1}\right)$, it follows that $\beta_{\hat{t}}^{1}\left(\hat{y}_{2}\right)=$ 
$\beta_{\hat{t}}^{1} \circ \beta_{\hat{t}}^{2}\left(\hat{y}_{1}\right) \preceq \beta_{\hat{t}}^{1} \circ \beta_{\hat{t}}^{2}\left(x_{1}^{*}\right)$. Consequently, when the condition in theorem 4 is satisfied, that is, $x_{1}^{*} \preceq \beta_{\hat{t}}^{1}\left(\hat{y}_{2}\right)$, the condition in theorem 2 is satisfied automatically, that is, $x_{1}^{*} \preceq \beta_{\hat{t}}^{1} \circ \beta_{\hat{t}}^{2}\left(x_{1}^{*}\right)$. Intuitively, the condition in theorem 2 evaluates the combined direct and indirect effects given by $\beta_{\hat{t}}^{1} \circ \beta_{\hat{t}}^{2}$ at $x_{1}^{*}$, and the condition in theorem 4 evaluates the combined effects at $\hat{y}_{1}$, which is higher than $x_{1}^{*}$.

The need for a stronger condition in multi-player games arises due to additional strategic interaction among the players. For example, consider a three-player game in which player 1 exhibits strategic substitutes and players 2 and 3 exhibit strategic complements. The natural generalization of the condition in theorem 2 would be: $x_{1}^{*} \preceq \beta_{\hat{t}}^{1}\left(\beta_{\hat{t}}^{2}\left(x_{-2}^{*}\right), \beta_{\hat{t}}^{3}\left(x_{-3}^{*}\right)\right)$. As shown in the Crime and Punishment-2 example above, this is not sufficient to guarantee monotone comparative statics. Intuitively, when the parameter increases from $t^{*}$ to $\hat{t}$, the direct effect on players 2 and 3 is captured by $\left(\beta_{\hat{t}}^{2}\left(x_{-2}^{*}\right), \beta_{\hat{t}}^{3}\left(x_{-3}^{*}\right)\right)$, which raises their strategies. But an increase for player 2 has a further impact for player 3, due to strategic complements, and vice-versa. The Crime and Punishment-2 example essentially shows that not including these additional effects may lead to an incorrect evaluation of the combined effects. The condition in theorem 4 adjusts for these effects by applying the combined evaluation on $\hat{y}_{-i}$, which is larger than $x_{-i}^{*}$.

A benefit of the condition in theorem 4 is that there are no restrictions on strategy spaces to be linearly ordered, as required by theorem 2 .

A similarity between theorem 4 and theorem 2 is that the condition needs to hold for players with strategic substitutes only. There is no additional restriction on players with strategic complements.

The following example shows an application of theorem 4 .

Example 5 (Cournot Oligopoly). Consider 3 firms competing in quantities. Firm 
1 is a large firm (or, say, an incumbent) that can produce one of three levels of output: Low, Medium, and High (denoted $L_{1}, M_{1}$, and $H_{1}$ ). It exhibits strategic substitutes. Firms 2 and 3 are smaller (or, say, potential entrants) and are capable of producing either Low or Medium level of output. Thus, $X_{1}=\left\{L_{1}, M_{1}, H_{1}\right\}, X_{2}=\left\{L_{2}, M_{2}\right\}$, and $X_{3}=\left\{L_{3}, M_{3}\right\}$. Suppose the smaller firms experience a technological spillover if enough output is produced by their rival firms, and therefore, each exhibits strategic complements. Payoffs are as follows.

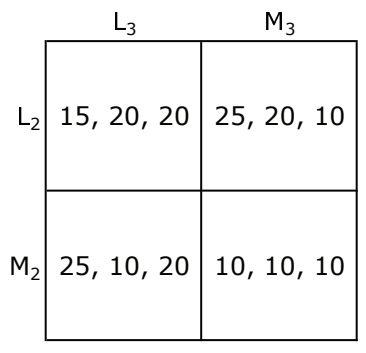

$\mathrm{L}_{1}$

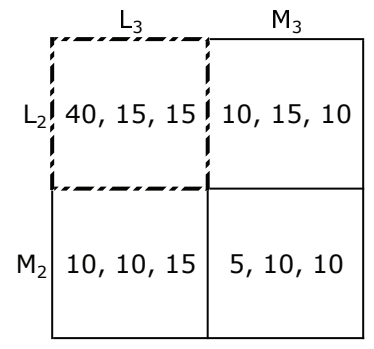

$M_{1}$

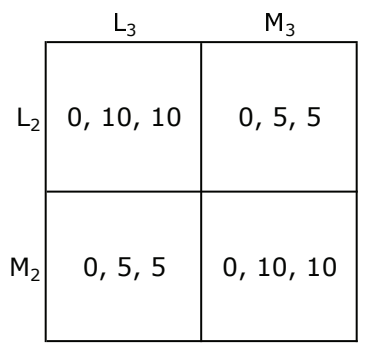

$\mathrm{H}_{1}$

Figure 7: Cournot Oligopoly, before parameter change

Notice that a smaller firm is only willing to produce the medium level of output, if both competitors produce their maximum levels. Also notice the strategic substitutes property of the large firm: it is only willing to produce a level other than $L_{1}$ if both competitors produce low levels. It is easy to check that the unique equilibrium is $x^{*}=\left(M_{1}, L_{2}, L_{3}\right)$. Now let the parameter $t$ increase to some $\hat{t}, \hat{t} \succ t^{*}$, and consider the following payoffs.

Notice that the parameter increase is (weakly) complementary for each firm. Firm 3 is more willing to increase its output: it will produce $M_{3}$ as long as one of its competitors is producing more than the low level of output. Firm 2 is now willing to produce $M_{2}$, if, and only if, firm 1 produces $H_{1}$. The condition in theorem 4 needs to be checked for firm 1 only (the firm with strategic substitutes). In this case, 


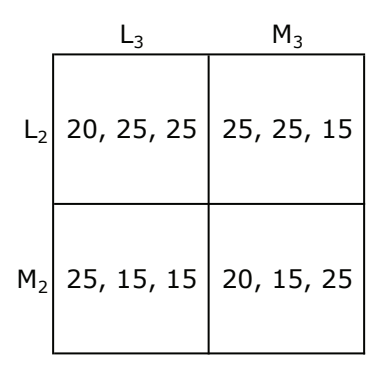

$\mathrm{L}_{1}$

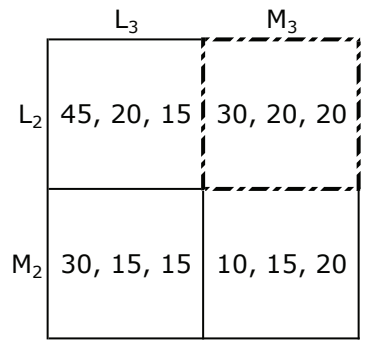

$M_{1}$

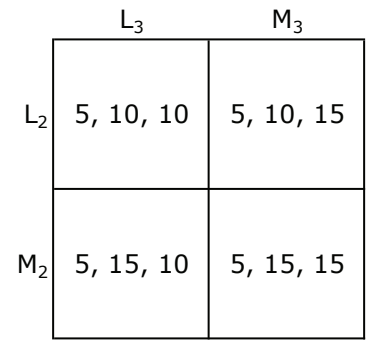

$\mathrm{H}_{1}$

Figure 8: Cournot Oligopoly, after parameter change

$\hat{y}_{1}=\beta_{\hat{t}}^{1}\left(x_{2}^{*}, x_{3}^{*}\right)=\beta_{\hat{t}}^{1}\left(L_{2}, L_{3}\right)=M_{1}, \hat{y}_{2}=\beta_{\hat{t}}^{2}\left(\hat{y}_{1}, \bar{x}_{3}\right)=\beta_{\hat{t}}^{2}\left(M_{1}, M_{3}\right)=L_{2}$, and $\hat{y}_{3}=$ $\beta_{\hat{t}}^{3}\left(\hat{y}_{1}, \bar{x}_{2}\right)=\beta_{\hat{t}}^{3}\left(M_{1}, M_{2}\right)=M_{3}$. Therefore, $x_{1}^{*}=M_{1} \preceq M_{1}=\beta_{\hat{t}}^{1}\left(L_{2}, M_{3}\right)=\beta_{\hat{t}}^{1}\left(\hat{y}_{-1}\right)$. Consequently, there is a higher equilibrium: $\hat{x}=\left(M_{1}, L_{2}, M_{3}\right)$.

The result in theorem 4 can be extended to best response correspondences, as follows. The intuition remains the same.

Theorem 5. Consider a parameterized lattice game in which players $1, \ldots, J$ have strategic substitutes, and players $J+1, \ldots, I$ have strategic complements and the strict single-crossing property in $\left(x_{i} ; t\right)$.

For every $t^{*} \prec \hat{t}$ and every $x^{*} \in \mathcal{E}\left(t^{*}\right)$, let $\hat{y}=\left(\hat{y}_{i}\right)_{i \in I}$ be defined as follows: $\hat{y}_{i}=$ $\bar{\beta}_{\hat{t}}^{i}\left(x_{-i}^{*}\right)$, for $i=1, \ldots, J$, and $\hat{y}_{i}=\bar{\beta}_{\hat{t}}^{i}\left(\left(\hat{y}_{j}\right)_{j=1}^{J} ;\left(\bar{x}_{j}\right)_{j=J+1, j \neq i}^{I}\right)$, for $i=J+1, \ldots, I$. If for $i=1, \ldots, J, x_{i}^{*} \preceq \underline{\beta}_{\hat{t}}^{i}\left(\hat{y}_{-i}\right)$, then there is $\hat{x} \in \mathcal{E}(\hat{t})$ such that $x^{*} \preceq \hat{x}$.

Proof. Notice that for every $i, x_{i}^{*} \preceq \hat{y}_{i}$, as follows. For $i=1, \ldots, J$, using single-crossing property, $x_{i}^{*} \preceq \bar{\beta}_{t^{*}}^{i}\left(x_{-i}^{*}\right) \preceq \bar{\beta}_{\hat{t}}^{i}\left(x_{-i}^{*}\right)=\hat{y}_{i}$. And for $i=J+1, \ldots, I$, $x_{i}^{*} \preceq \bar{\beta}_{t^{*}}^{i}\left(x_{-i}^{*}\right) \preceq \bar{\beta}_{t^{*}}^{i}\left(\left(\hat{y}_{j}\right)_{j=1}^{J} ;\left(\bar{x}_{j}\right)_{j=J+1, j \neq i}^{I}\right) \preceq \bar{\beta}_{\hat{t}}^{i}\left(\left(\hat{y}_{j}\right)_{j=1}^{J} ;\left(\bar{x}_{j}\right)_{j=J+1, j \neq i}^{I}\right)=\hat{y}_{i}$, where the second inequality follows from strategic complements, and the last inequality from single-crossing property.

For $i=1, \ldots, I$, let $B_{i}=\left[x_{i}^{*}, \hat{y}_{i}\right]$, and let $B=\times_{i=1}^{I} B_{i}$. For $i=1, \ldots, I$, consider $\beta_{\hat{t}}^{i}$ 
on $B_{-i}$. Then $x_{i}^{*} \preceq \underline{\beta}_{\hat{t}}^{i}\left(\hat{y}_{-i}\right)$ by assumption, and $\bar{\beta}_{\hat{t}}^{i}\left(x_{-i}^{*}\right)=\hat{y}_{i}$, by definition. Therefore, $\beta_{\hat{t}}^{i}\left(B_{-i}\right) \subseteq B_{i}$; that is, $\beta_{\hat{t}}^{i}$ restricted to $B_{-i}$ maps into $B_{i}$. Similarly, for $i=J+1, \ldots, I$,

consider $\beta_{\hat{t}}^{i}$ on $B_{-i}$. Strict single-crossing property in $\left(x_{i} ; t\right)$ yields $x_{i}^{*} \preceq \bar{\beta}_{t^{*}}^{i}\left(x_{-i}^{*}\right) \preceq$ $\underline{\beta}_{\hat{t}}^{i}\left(x_{-i}^{*}\right)$. Moreover, $\beta_{\hat{t}}^{i}\left(\hat{y}_{-i}\right)=\beta_{\hat{t}}^{i}\left(\left(\hat{y}_{j=1}^{J}\right) ;\left(\hat{y}_{j}\right)_{j=J+1, j \neq i}^{I}\right) \sqsubseteq_{i n} \beta_{\hat{t}}^{i}\left(\left(\hat{y}_{j=1}^{J}\right) ;\left(\bar{x}_{j}\right)_{j=J+1, j \neq i}^{I}\right)$, where the induced set order inequality follows from strategic complements. Therefore, $\bar{\beta}_{\hat{t}}^{i}\left(\hat{y}_{-i}\right) \preceq \bar{\beta}_{\hat{t}}^{i}\left(\left(\hat{y}_{j=1}^{J}\right) ;\left(\bar{x}_{j}\right)_{j=J+1, j \neq i}^{I}\right)=\hat{y}_{i}$. Thus, $\beta_{\hat{t}}^{i}\left(B_{-i}\right) \subseteq B_{i}$. Consequently, the joint best response correspondence satisfies $\beta_{\hat{t}}(B) \subseteq B$; that is, the restriction of $\beta$ to $B$ is a self-map, and applying Kakutani-Fan-Glicksberg's theorem, there is a fixed point $\hat{x} \in \mathcal{E}(\hat{t})$ such that $x^{*} \preceq \hat{x}$.

\section{Conclusion}

This paper focuses on games with both strategic substitutes and strategic complements. In general, such games may behave differently when compared to either GSC or GSS.

We show that in games with both strategic substitutes and complements, equilibria do not decrease as the parameter increases. Moreover, using newer techniques, we provide conditions that guarantee that an increase in the parameter leads to an increase in the equilibrium; in other words, conditions under which monotone comparative statics is guaranteed.

For two-player games in which one player exhibits strategic substitutes, the other player exhibits strategic complements, and each player has a linearly ordered strategy space, we characterize monotone comparative statics via a condition on the best response of only the player with strategic substitutes. (No additional condition is imposed on the player with strategic complements.) The condition is intuitive and is based on a trade-off between the direct parameter effect and the indirect strategic 
substitute effect.

This characterization does not hold more generally: either for two-player games with more general strategy spaces, or for games with more players, as shown in several examples. In this regard, games with both strategic substitutes and complements behave differently from GSS.

For more general cases, we present sufficient conditions that guarantee monotone comparative statics. As in the two-player case, these conditions are needed only for players with strategic substitutes. The conditions are stronger than in the two-player case, but still involve a trade-off between the direct parameter effect and the indirect strategic substitute effect. 


\section{References}

Acemoglu, D., And M. K. Jensen (2009): "Aggregate comparative statics," Working Paper, Department of Economics, University of Birmingham and MIT.

(2010): "Robust comparative statics in large static games," Working Paper, Department of Economics, University of Birmingham.

Amir, R. (1996): "Cournot Oligopoly and the Theory of Supermodular Games," Games and Economic Behavior, 15, 132-148.

Amir, R., F. Garcia, And M. KnaufF (2010): "Symmetry-breaking in two-player games via strategic substitutes and diagonal nonconcavity: a synthesis," Journal of Economic Theory, 145(5), 1968-1986.

Amir, R., And V. E. Lambson (2000): "On the Effects of Entry in Cournot Markets," The Review of Economic Studies, 67(2), 235-254.

Becker, G. S. (1968): "Crime and Punishment: An Economic Approach," Journal of Political Economy, 76, 169-217.

Bulow, J. I., J. D. Geanakoplos, and P. D. Klemperer (1985): "Multimarket Oligopoly: Strategic Substitutes and Complements," Journal of Political Economy, 93(3), 488-511.

Dixit, A. (1987): "Strategic behavior in contests," American Economic Review, $77(5), 891-898$.

ECHEnique, F. (2002): "Comparative statics by adaptive dynamics and the correspondence principle," Econometrica, 70(2), 257-289.

(2004): "A characterization of strategic complementarities," Games and Economic Behavior, 46(2), 325-347.

Edlin, A., And C. Shannon (1998): "Strict Monotonicity in Comparative Statics," Journal of Economic Theory, 81(1), 201-219.

FudenberG, D., And J. Tirole (1984): "The fat-cat effect, the puppy-dog ploy, and the lean and hungry look," American Economic Review, 74(2), 361-366.

Jensen, M. K. (2010): "Aggregative Games and Best-Reply Potentials," Economic Theory, 43(1), 45-66.

Lippman, S. A., J. W. Mamer, and K. F. McCardle (1987): "Comparative Statics in non-cooperative games via transfinitely iterated play," Journal of Economic Theory, 41(2), 288-303. 
Milgrom, P., And J. Roberts (1990): "Rationalizability, learning, and equilibrium in games with strategic complementarities," Econometrica, 58(6), 1255-1277. 459.

Milgrom, P., and C. Shannon (1994): "Monotone Comparative Statics," Econometrica, 62(1), 157-180.

Monaco, A., and T. SABARWAL (2011): "A non-robustness in the order structure of the equilibrium set in lattice games," Working paper, Department of Economics, University of Kansas.

QuAH, J. K.-H. (2007): "The Comparative Statics of Constrained Optimization Problems," Econometrica, 75(2), 401-431.

Quah, J. K.-H., And B. Strulovici (2009): "Comparative statics, informativeness, and the interval dominance order," Econometrica, 77(6), 1949-1992.

Roy, S., and T. Sabarwal (2008): "On the (Non-)Lattice Structure of the Equilibrium Set in Games With Strategic Substitutes," Economic Theory, 37(1), 161-169.

- (2010): "Monotone comparative statics for games with strategic substitutes," Journal of Mathematical Economics, 46(5), 793-806.

- (2012): "Characterizing stability properties in games with strategic substitutes," Games and Economic Behavior, 75(1), 337-353.

Schipper, B. C. (2003): "Submodularity and the evolution of Walrasian behavior," International Journal of Game Theory, 32, 471-477.

Shannon, C. (1995): "Weak and Strong Monotone Comparative Statics," Economic Theory, 5(2), 209-227.

Singh, N., ANd X. Vives (1984): "Price and quantity competition in a differentiated duopoly," Rand Journal of Economics, 13(4), 546-554.

Sobel, J. (1988): "Isotone comparative statics in supermodular games," Mimeo. SUNY at Stony Brook.

TombaK, M. M. (2006): "Strategic Asymmetry," Journal of Economic Behavior and Organization, 61(3), 339-350.

Topkis, D. (1978): "Minimizing a submodular function on a lattice," Operations Research, 26, 305-321.

(1979): "Equilibrium points in nonzero-sum $n$-person submodular games," SIAM Journal on Control and Optimization, 17(6), 773-787. 
- (1998): Supermodularity and Complementarity. Princeton University Press.

Villas-Boas, J. M. (1997): "Comparative Statics of Fixed Points," Journal of Economic Theory, 73(1), 183-198.

Vives, X. (1990): "Nash Equilibrium with Strategic Complementarities," Journal of Mathematical Economics, 19(3), 305-321.

— (1999): Oligopoly Pricing. MIT Press.

(2005): "Complementarities and Games: New Developments," Journal of Economic Literature, 43(2), 437-479.

Zhou, L. (1994): "The Set of Nash Equilibria of a Supermodular Game is a Complete Lattice," Games and Economic Behavior, 7(2), 295-300.

Zimper, A. (2007): "A fixed point characterization of the dominance-solvability of lattice games with strategic substitutes," International Journal of Game Theory, 36(1), 107-117. 